\section{Is the Martian core solid or liquid?}

from Peter J. Smith

From the mass, radius and moment of inertia of Mars as determined by orbiting and passing spacecraft, it may be reasonably concluded that the planet has a central core. But is the core solid or liquid? The correct answer to this question is not only of interest in its own right; it may also help to resolve a disagreement which is about to arise over whether or not Mars possesses an internal magnetic field. Last year, Dolginov et al. (J. geophys. Res., 78, 4779; 1973) interpreted Mars 2 and Mars 3 magnetometer data in terms of just such a field; but apparently Bogdanov and Vaisberg will shortly argue that the observed magnetic field variations are the result of solar windplanetary ionosphere interaction instead. Clearly, if it could be shown that the Martian core is solid, an intrinsic magnetic field (at least, produced by dynamo action) would be ruled out, although it must be admitted that proof of a liquid core would fail to resolve the issue either way.

In a situation where the confirmed presence or absence of an internal magnetic field cannot itself be used to infer the state of the core, the obvious approach is to examine the thermal conditions in the Martian interior. Unfortunately, there are no experimental thermal data from Mars. Mariner 9 revealed evidence of extensive volcanism (including a series of prominent shield volcanoes), mildly cratered plains similar to the lunar maria, and circular features such as domes and craters; but although this suggests that at some time in the past Mars must have been hot enough for partial melting to have occurred at depth, it can give no sure indication of present conditions.

In an attempt to deduce whether the Martian core is solid or liquid, Young and Schubert (Geophys. Res. Lett., 1, 157 ; 1974) have therefore constructed a thermal model of Mars which involves no experimentally determined thermal boundary conditions but draws on experience and information gained from the application of similar techniques to the Earth. Indeed, as far as structure is concerned the model is a straight 'Earth model' with core, mantle and crust separated by rigid boundaries. The core itself is assumed to contain no heat sources (so there is zero heat flux at the core-mantle boundary), but heat sources are distributed uniformly throughout the rest of the planet (so temperature and thermal gradients are continuous across the crust-mantle boundary). The crust, whose outer boundary is taken to be an isothermal surface, is only able to conduct heat; but the mantle is a region in which solid-state thermal convection is an important mode of heat transfer. It is this last characteristic which particularly distinguishes the Young-Schubert model from most previous thermal model studies of planetary interiors.

In numerical terms, two sets of core parameters are examined: an iron core with a radius 0.4 of the total Martian radius and mass 0.1 of the Martian mass, and a pure $\mathrm{FeS}$ core of radius 0.6 and mass 0.26 . These core models are the maximum density-minimum size and maximum size-minimum density cores consistent with observed mass, radius and moment of inertia of Mars. The radioactive heat source concentration in the crust-mantle is taken to be the same as that in the terrestrial mantle $\left(2.61 \times 10^{-7} \mathrm{erg} \mathrm{cm}^{-3} \mathrm{~s}\right)$. The thickness of the conducting crust will be determined by the depth at which the conduction solution for temperature rises to about $1,000^{\circ} \mathrm{C}$ above the surface temperature of the planet, and is thus highly dependent on the chosen value of heat source concentration. But crustal thickness should be at least $100 \mathrm{~km}$ because a thinner crust would probably be subject to plate tectonic activity, whereas no evidence of such activity is available from the Mariner 9 investigations.

The most important point to emerge from the model calculations is that the thermal convection in the mantle is remarkably successful in reducing temperatures (as in the Earth), thus vindicating its inclusion in the model in the first place. For a pure iron core, for example, a temperature difference of $8,000^{\circ} \mathrm{C}$ between the core-mantle boundary and the planetary surface when there is no convection is reduced to about $2,300^{\circ} \mathrm{C}$ when convection occurs at a Rayleigh number of 100 times the critical value for the onset of convection. The actual Rayleigh number is, of course, uncertain because viscosities are not known; but one certain point arising from the models is that, for pure conduction, temperatures rise above typical silicate melting temperatures. The implication here, therefore, is that convection in the Martian mantle is actually necessary to prevent largescale melting there.

And because viscosities are unknown, it is not possible to deduce absolutely whether the Martian core is solid or liquid. But what can be said is that if viscosity is lower than $10^{22}-10^{23} \mathrm{~cm}^{2} \mathrm{~s}^{-1}$ at temperatures above about $1,500^{\circ} \mathrm{C}$, mantle convection will then be efficient enough to cool the core-mantle boundary to temperatures below those at which $\mathrm{Fe}$ or $\mathrm{Fe}-\mathrm{FeS}$ cores would be liquid. As several workers have concluded that Martian viscosity is indeed below this limit, the Young-Schubert model suggests that a dynamo-produced magnetic field in the Martian core is not possible.

Finally, one other important conclusion to be drawn (specifically from the model temperature profiles) is that if partial melting occurs at all in the mantle it will do so at depths of about $300 \mathrm{~km}$. By analogy with the Earth, this suggests that the Martian lithosphere would be several hundred kilometres thick, or intermediate in thickness between those of the Earth and the Moon.

\section{Gravitational waves from collapsing stars}

from $P . C . W$. Davies

Although it is becoming increasingly likely that Professor Joseph Weber's longstanding attempt to detect gravitational waves from a cosmic source has not after all met with the spectacular success originally claimed, the stimulus that his work and propaganda has given to theoretical studies of this phenomenon continues to operate unabated. Gravitational waves are predicted to occur whenever massive objects are disturbed, much as electromagnetic radiation is produced by accelerating charged particles. The main difficulty in the detection of the former rather than the latter is the incredibly low cross section of ordinary matter to these disturbances. Consequently, only very massive and violent cosmic events occurring in relative proximity to the Earth are capable of producing a pulse of gravitational radiation sufficiently energetic to be detectable with current technology.

Attention has traditionally been given to three possible cosmic sources of such radiation: violent agitations occurring in the primaeval fireball of the big bang, objects crashing into black holes and the explosion or collapse of stars. Most books on gravitation contain order of magnitude estimates of the strength of the ensuing radiation, but few explicit numerical calculations seem to have been published. A welcome contribution to the physics of gravitational wave pulses from collapsing stars appears in the August 1 issue of Astrophysical Journal (191, L105L107; 1974). Detailed calculations have been performed by Thuan and Ostriker of the Princeton University Observatory for a number of simple scenarios of the collapse of a white dwarf star into a neutron star or black hole.

It is well known that there can be no gravitational radiation from a spherically symmetrical collapsing object. A real star will, however, always possess asymmetries, and these tend to become amplified during the collapse process. 